\title{
Tagging of Isobars using Energy Loss and Time-of-Flight Measurements
}

\author{
D. Shapira, T. A. Lewis, and P. E. Mueller
}

\begin{abstract}
-
The technique for tagging isobars in a mixed beam by measuring energy loss by time-of-flight has been tested. With this method, isobar separation should improve by allowing more energy loss (thicker absorber), but only if one can control absorber homogeneity. Measurements of beam energy loss and energy spread obtained under such conditions were shown to be close to predicted values using both collisional and charge exchange contributions to energy straggling. The calculation of energy straggling allows us to study the efficacy of this method for isobar separation when applied to different mass ranges and beam energies. Separation in a most difficult case, an analyzed beam of $A=132$ isobars at energies near $3 \mathrm{MeV} / \mathrm{A}$ has been demonstrated. The time-of-flight information can be added on line as an additional tag to the data stream for events of interest. Such event by event tagging enables one to study the effect of differences in isobaric mixture in the beam on the reaction outcome even when isobar separation is not complete.
\end{abstract}

\section{INTRODUCTION}

In general, one may expect isobaric contaminants to be present at some level in the beams delivered at radioactive ion beam (RIB) facilities. This may be due to the inherent method of beam delivery, for example, a mixture of beams with similar mass-to-charge ratios from a fragmentation source or isobars that escape separation in beams delivered from an ISOL (Isotope Separation On Line) source. In the next generation of RIB facilities, where deceleration and re-acceleration of RIBs with very short lifetimes (lms) is contemplated, decay-in-flight may also introduce significant amounts of isobaric contamination. Since isobars are very close in mass, their separation is not an easy task.

When isobar masses are separated by a small fraction of their total mass $(\leq 1 / 20000)$, separation via momentum (magnetic spectrograph) or time-of-flight (TOF) measurements is extremely difficult. Isobars are most easily separated by exploiting their difference in nuclear charge $(z)$. The combination of a passive absorber and a TOF measurement can be used to measure energy loss and determine the nuclear charge. The TOF detectors can handle rates up to several $\mathrm{MHz}$. This method can be applied with different degrees of success depending on the energy and mass of the beams under investigation. It is particularly difficult for heavy and medium mass nuclei at energies below $6 \mathrm{MeV} / \mathrm{A}$ such as are contemplated for many ISOL-based RIB facilities planned or under construction world wide. By using a specially designed gas-filled cell as absorber material, we were able to keep absorber inhomogeneity below $0.5 \%$. We can predict the spread in beam energy as a function of nuclear charge, mass, energy, and energy loss. In this paper we compare predictions of resolving power with this method to measurements

D. Shapira and P. E. Mueller are members of the Physics Division and T. A. Lewis is a member of the Engineering Science and Technology Division at the Oak Ridge National Laboratory, Oak Ridge, Tennessee37831, USA.

Oak Ridge National Laboratory is managed by UT-Battelle, LLC under contract DE-AC05-00OR22725 for the U. S. Department of Energy. in several cases. Limits to this method's applicability are discussed and a successful application of this method to the most difficult task of tagging $\mathrm{A}=132$ isobars is demonstrated.

\section{DesCRIPTION OF MethoD}

Ions, with kinetic energies below $1 \mathrm{GeV} /$ nucleon, passing through an absorber lose energy mostly through repeated ionization of the medium's atoms. This electronic energy loss is approximated by the Bethe-Bloch formula. Projectile dependence in this formula is through the square of the nuclear charge (z) and kinetic energy [I]. Therefore, the fractional difference in energy loss suffered by two neighboring isobars moving at the same velocity is given approximately by $2 / z$. If the energy loss suffered by the isobars passing the same absorber is a significant fraction of the ion's total energy $(0.1 \leq \Delta E / E \leq 0.5)$, the difference in isobar energies as they emerge from the absorber will also be significant and detectable. The situation, though, is complicated due to spread in the ion's energy as it passes through the absorber. The spread is caused by energy loss straggling and by inhomogeneities in absorber thickness. The difference in energy losses suffered by neighboring isobars, which varies linearly with energy loss, will eventually become larger than the spread caused by energy straggling, which is proportional to the square root of the energy loss. When planning to apply this method, one needs reliable estimates for energy loss and energy straggling of the isobars in the absorber medium of choice. Energy loss estimates presented here, made with the code SRIM [2], were shown to be reliable in all our tests. The energy straggling predicted by SRIM, however, turned out to be much smaller than the values we measured for all particles heavier than $A=4$. A recipe to calculate energy straggling for heavy ions that includes contributions from collisional recoil and ioncharge fluctuations provided more accurate results [3].

\section{Measuring Energy Loss AND EnERgy SPREAD fOR Heavy Ions Passing through Absorbers}

Before attempting to tag isobars in a mixed radioactive beam, we had to evaluate the assumptions made for beam energy spread in the absorber. Reliable estimates of beam energy spread as a function of beam energy loss are most critical for planning isobar separation with this technique. When considering the use of this technique for beam tagging, we also need to consider the loss in beam intensity due to multiple scattering in the absorber. The beam can spread to a very large size if it is not refocussed. Requirements that will render this method for isobar separation viable are as follows:

. The isobars must experience substantial energy loss (absorber material and thickness).

. The uncertainty in post-absorber beam energy must be mini- 


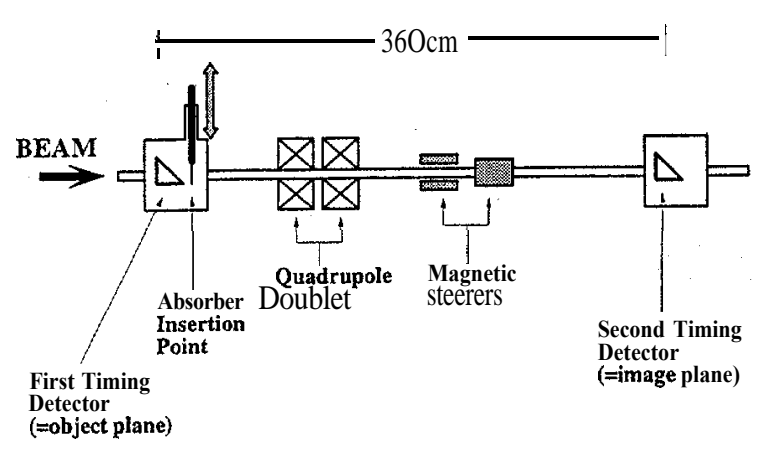

Fig. 1. Beamline setup for studying isobar tagging.

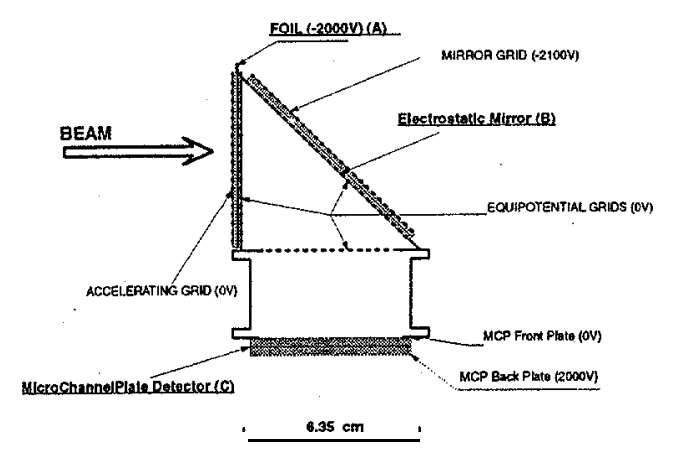

Fig. 2. Components of the timing detector - a sketch.

mized (absorber homogeneity).

. The TOF measurements must be optimized (instrumentation). . The beam transmission must be optimized (beam focussing).

A test beamline with two timing detectors, and one quadrupole doublet and two magnetic steerers between them, was used to measure the TOF of beam particles. Fig. 1 shows a schematic layout of the beamline that we used. It shows the location of a mechanism for inserting different absorbers at a close distance following the first timing detector. Two timing detectors (see Ref. [7]) as shown in Fig. 2 were employed.

\section{A. First test with ${ }^{58} \mathrm{Ni}$ beam}

Radioactive ${ }^{56} \mathrm{Ni}$ beam will be produced at the Holifield Radioactive Ion Beam Facility (HRIBF) at Oak Ridge National Laboratory (ORNL) with a batch mode ion source where ${ }^{56} \mathrm{Ni}$ is sputtered from a ${ }^{58} \mathrm{Ni}$ target that has been previously bombarded with an intense proton beam for several hours [4]. This ion source will also produce copious amounts of ${ }^{56} \mathrm{Co}$ that will be hard to separate from ${ }^{56} \mathrm{Ni}$. As a result, a mixed beam of ${ }^{56} \mathrm{Ni}$ and ${ }^{56} \mathrm{Co}$ will reach the target. It will be impossible without tagging to determine whether detected evaporation residues or fission products result from collisions between ${ }^{56} \mathrm{Co}$ or ${ }^{56} \mathrm{Ni}$ with the target nucleus. If we were able to tag the beam before it reaches the target and provide this information every time a fu-

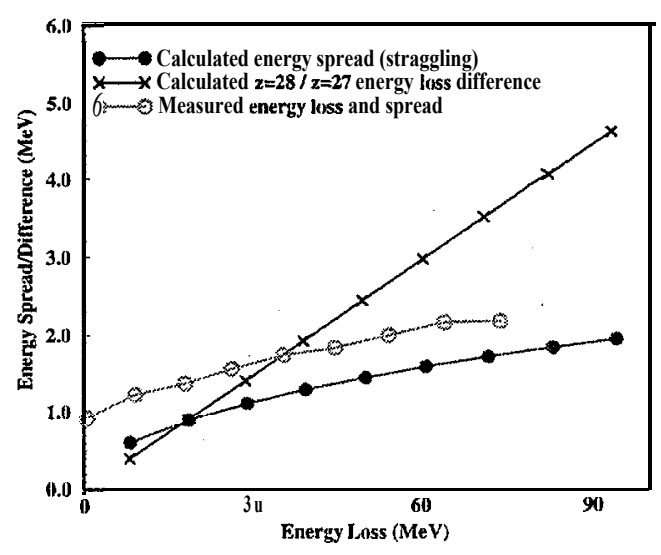

Fig. 3. Measured and calculated energy spread for $270 \mathrm{MeV}^{58} \mathrm{Ni}$ passing through the gas cell and the anticipated separation for $z=27$ and $z=28$ isobars.

sion or fission product is detected, we could determine whether the process originated from reactions with ${ }^{56} \mathrm{Ni}$ or ${ }^{56} \mathrm{Co}$.

Several TOF measurements for a $235 \mathrm{MeV}{ }^{58} \mathrm{Ni}$ test beam were made with the two timing detectors and several different absorber foil combinations inserted into the beamline. The absorber foils we used were made of Mylar and polypropylene (with thickness ranging between 1 and $15 \mu \mathrm{m}$ ). The results of several such measurements demonstrated that the inhomogeneity in foil thickness would be a serious problem.

\section{B. Building a homogeneous absorber - second test with ${ }^{58} \mathrm{Ni}$}

We have decided to use a gas cell as a means to achieve a high degree of uniformity in the beam energy degrader thickness. A $7 \mathrm{~cm}$ long cylindrical gas cell with thin $(0.9 \mu \mathrm{m})$ entrance and exit windows was built. The absorber gas chosen, isobutane $\left(\mathrm{C}_{4} \mathrm{H}_{10}\right)$, has relatively low nuclear charges but is a relatively heavy molecule.

We performed measurements with $270 \mathrm{MeV}^{58} \mathrm{Ni}$ using different amounts of gas in the cell to vary the amount of energy loss in the absorber. Fig. 3 shows measured energy spread in the beam as a function of measured energy loss. It also shows predictions of energy spread. The measured and predicted energy spreads show the same trend (square root of energy loss). The zero offset (about $0.92 \mathrm{MeV}$ at zero energy loss) is attributed to instrumental TOF resolution of $210 \mathrm{ps}$. From the data presented in Fig. 3, it is clear that allowing for energy loss of $30 \%$ of the total energy would suffice to separate ${ }^{56} \mathrm{Ni}$ and ${ }^{56} \mathrm{Co}$ when the need arises. Table I lists the relevant quantities after allowing for $90 \mathrm{MeV}$ energy loss of ${ }^{58} \mathrm{Ni}$ in the absorber.

TABLE I

DEGRADATION IN BEAM QUALITY FOR IONS PASSING THROUGH AN ABSORBERMATERIAL.

\begin{tabular}{|c|c|c|c|c|}
\hline Isobar & E-beam & E-loss & E-straggling & E-difference \\
\hline mass & $\mathrm{MeV}$ & $\mathrm{MeV}$ & $\mathrm{MeV}$ & $\mathrm{MeV}$ \\
\hline $\mathrm{A}=58$ & 270 & 90 & 2.00 & 4.50 \\
$\mathrm{~A}=17$ & 40 & 20 & 0.37 & 3.68 \\
\hline
\end{tabular}




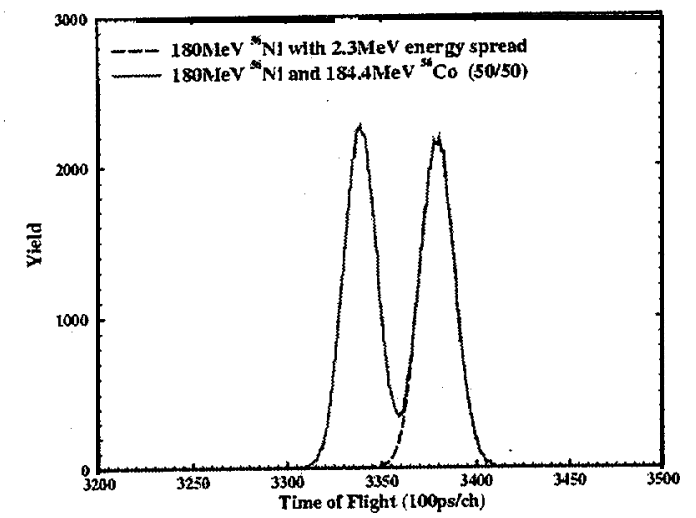

Fig, 4. Simulated time-of-flight spectra for ${ }^{56} \mathrm{Ni}$ and a mixture of ${ }^{56} \mathrm{Ni}$ and ${ }^{56} \mathrm{Co}$.

Fig. 4 shows the time-of-flight spectrum that can be expected for a 1:1 mixture of $270 \mathrm{MeV}{ }^{56} \mathrm{Ni}$ and ${ }^{56} \mathrm{Co}$ allowing for $90 \mathrm{MeV}$ energy loss in the absorber (see Fig. 3). The degree of separation expected is shown in Fig. 4. It shows the TOF spectra for ${ }^{56} \mathrm{Ni}$ alone and for the mix of ${ }^{56} \mathrm{Ni}$ and ${ }^{56} \mathrm{Co}$. The detector resolution assumed in our simulation was about $310 \mathrm{ps}$ and is much lower than the 2.8 ns FWHM seen in Fig. 4. Simulations under different conditions have shown that the main culprit causing the observed spread in time-of-flight is energy straggling in the absorber. The remedy is to allow more energy loss in the absorber that will bring about an increase in the energy difference betwee ${ }_{-}^{56} \mathrm{Ni}$ and ${ }^{56} \mathrm{Co}$. The expected increase in energy straggling should occur at a lower rate. The same simulation also showed that the transmission from the first timing detector to the second is better than $50 \%$ for beam sizes varying from $3 \mathrm{~mm}$ to $10 \mathrm{~mm}$ in diameter (allowing no increase in beam diameter size at the image plane). We define the transmission efficiency as the ratio of the number of hits at the image plane that fall within the area bound by the object size to the total number of particles launched. With this definition, the transmission without the beam focussing elements would have been about $0.2 \%$ and $2.0 \%$ for beam size diameters of $3 \mathrm{~mm}$ and 10 $\mathrm{mm}$, respectively.

\section{Separation of ${ }^{17} \mathrm{~F}$ and ${ }^{17} \mathrm{O}$}

Nuclear reactions between light $(\mathrm{A}<40)$ radioactive beams and light targets are of interest to nuclear astrophysicists because they may represent nuclear processes taking place in the hot interiors of exploding stars. Several of these studies were conducted at HRIBF with radioactive ${ }^{17} \mathrm{~F}$ beams at energies ranging between 15 to $33 \mathrm{MeV}$ [5], [6]. The RIB ion source produces ${ }^{17} \mathrm{~F}$ along with a copious amount of ${ }^{17} \mathrm{O}$. When coincidence detection of binary products is feasible, contributions from different beam species can be unfolded, but with low intensity radioactive beams this luxury is not always afforded. In thick target experiments [6], we made sure that only ${ }^{17} \mathrm{~F}$ arrives at the experiment by stripping the ion beam at the end of the acceleration cycle and tuning the final analyzing magnet to allow only charge state $9^{+}$to go through, thus ensuring complete re-

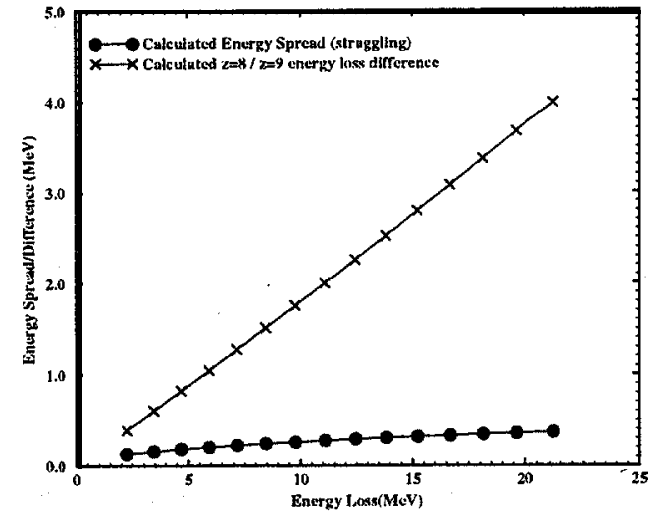

Fig. $5.40 \mathrm{MeV}^{17} \mathrm{~F}$ energy straggling and difference in energy loss of nearest isobar ${ }^{17} \mathrm{O}$ as a function of total energy loss.

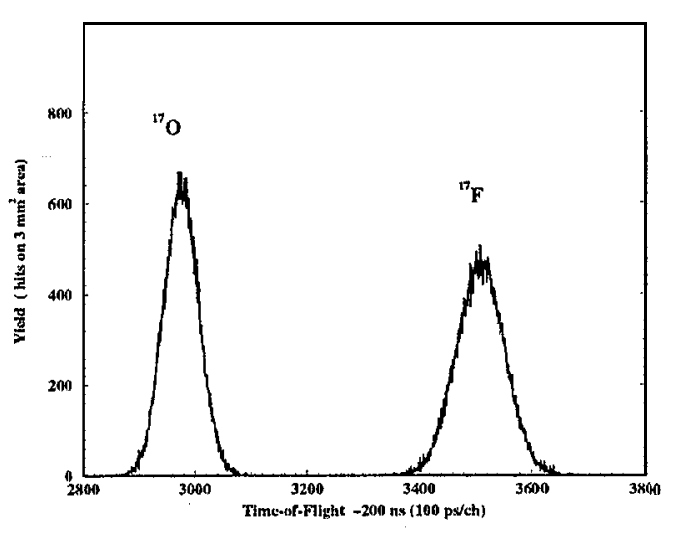

Fig. 6. Simulated time-of-flight spectra for a 1: 1 mixture containing ${ }^{17} \mathrm{~F}$ and ${ }^{17} \mathrm{O}$, initial energy $40 \mathrm{MeV}, \mathrm{AE}=20 \mathrm{MeV}$.

jection of ${ }^{17} \mathrm{O}$. At $20 \mathrm{MeV}^{17} \mathrm{~F}$, however, only $3 \%$ of the beam will be fully stripped, i.e., a $97 \%$ loss in beam intensity ensues. The method of isobar tagging discussed here could become an attractive alternative at such low energies.

Fig. 5 shows predictions of energy straggling for $40 \mathrm{MeV}^{17} \mathrm{~F}$ passing through the gas absorber, as a function of energy loss. Also shown is the difference in energy between ${ }^{17} \mathrm{~F}$ and ${ }^{17} \mathrm{O}$ that had the same initial energy and passed through the same absorber. It is obvious that separation of ${ }^{17} \mathrm{~F}$ and ${ }^{17} \mathrm{O}$ will be an easy task.

The simulated time-of-flight spectrum for a $1: 1$ mixture of ${ }^{17} \mathrm{~F}$ and ${ }^{17} \mathrm{O}$ is shown in Fig. 6. It shows that complete separation of $20 \mathrm{MeV}^{17} \mathrm{~F}$ is possible on an event by event basis. The relevant quantities after allowing for approximately $20 \mathrm{MeV}$ energy loss for the $\mathrm{A}=17$ mixture are listed in Table I. The incident beam and object size, in this case, are bounded by a 3-mm diameter circle. The overall transmission predicted in our simulation, which incorporates fluctuations in beam energy, charge, and angular spread, is still near 50\% (and higher if we allow larger image sizes). The main contribution to the predicted spread in time-of-flight is from the $370 \mathrm{keV}$ spread in the ion's energy due 


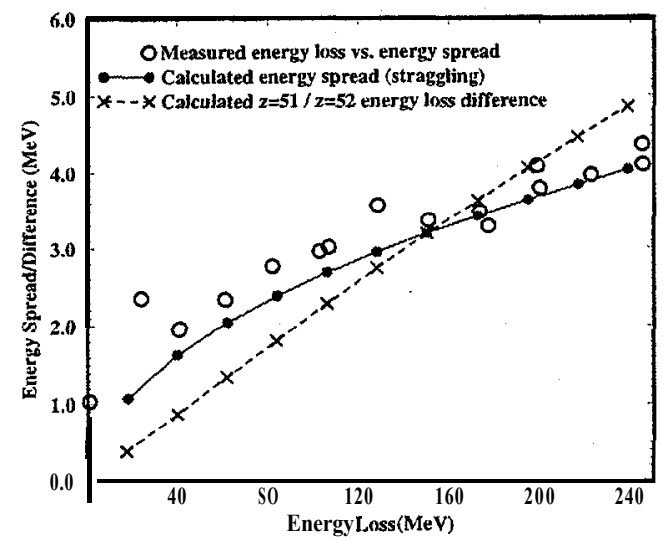

Fig. 7. Measured and calculated energy spread for $450 \mathrm{MeV}^{132} \mathrm{Te}$ passing through the gas cell and the anticipated separation for $z=51$ and $z=52$ isobars.

to energy straggling in the absorber.

Measurements presented in this report show that we can use SRIM [2] and formulae from GEANT [3] to make predictions about energy loss and energy spread for ions passing through an absorber medium quite reliably. This information combined with the detector system's time resolution and properties of the beamline allows us to predict the efficacy of using energy loss data to tag isobar mixtures in the beam. The next section shows the results from a case where this method was put through a most stringent test.

\section{IsObar SEPARATION IN A MASS 132 MiXTURe}

The beam of mass $A=132$ produced in the HRIBF fission source is known to contain a mixture of several isobars [8]. The beamline leading from the source to the post-acce!erator is equipped with a large magnet designed to help with isobar separation. We have decided to try the technique of energy loss measurements to test if we can tag isobars in the accelerated beam. Such measurements could help us obtain immediate feedback on the effects of tuning the isobar separator magnet and also test our tagging technique for future experiments.

To measure the effectiveness of this method for $\mathrm{A}=132$ isobars, we first measured the energy spread as a function of energy loss for a radioactive pure ${ }^{132} \mathrm{Te}$ beam. The beam was accelerated to $450 \mathrm{MeV}$ and analyzed in the test beamline (see Fig. 1). The results of these measurements are shown in Fig. 7.

The calculated energy spread agrees very well with the measured energy spread of the actual beam when the gas cell is used as an absorber. The first point without any absorber shows a width of $1 \mathrm{MeV}(0.2 \%$ in energy) attributable to detector timing resolution (180 ps). The energy spread of the second data point at $18 \mathrm{MeV}$ energy loss is due to the entrance and exit foil on the gas cell. As repeated measurements with foils alone have shown, the energy spread of particles passing a foil often surpasses what is expected from energy straggling in the foil. This, we assume, is due to inhomogeneities in the foil. Note that the third data point shows a decrease in energy spread. This may be due to the stretching of the foil by the gas filling the cell. Once

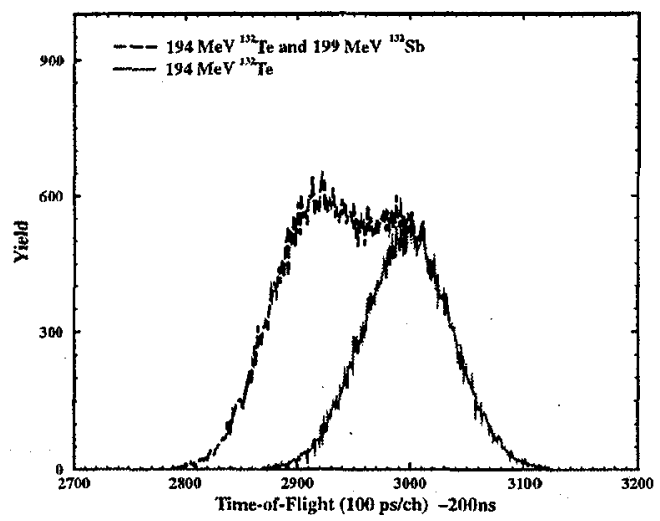

Fig. 8. Simulated TOF spectra for $450 \mathrm{MeV}{ }^{132} \mathrm{Te}$ and ${ }^{132}$ Sbpassing through the gas cell containing 100 torr isobutane gas.

the magnitude of the energy loss is dominated by losses in the gas absorber, the measurements tend to agree with the predictions of energy spread due to straggling.

From the data in Fig. 7, it is clear that for this case of relatively low energy $A=132$ isobar mixture we can not expect complete separation. It was important though to see if the method works at the level predicted and if tagging is a viable option.

We then used the predicted energy losses of different isobars and the measured energy spread as seen in Fig. 7 to simulate the expected separation for ${ }^{132} \mathrm{Te}$ and ${ }^{132} \mathrm{Sb}$ isobars. The results of these simulations are shown in Fig. 8 and confirm that there is substantial overlap but one can attain some degree of isobar separation. These TOF data, when recorded for each event of interest in the reaction, provide an additional variable (tag) associating reaction outcome with differences in isobar mixtures in the beam. Such a spectrum could also provide immediate feedback when one tries alternative beam tuning in order to enhance the yield of one particular isobar.

The beam of $A=132$ products was then analyzed in our test beamline and Fig. 9 shows several TOF spectra measured for beam particles that passed the gas cell filled with 100 ton isobutane gas. Several attempts to block out the ${ }^{132} \mathrm{Te}$ component in the beam and modify the ratio of ${ }^{132} \mathrm{Sb}$ and ${ }^{132} \mathrm{Sn}$ were made by modifying the beam tune through the low energy section of the beamline. Fig. 9 shows the result of different current settings in the second stage magnet (isobar separator) in the low energy section. The different time-of-flight spectra show that the isobar mixture can be controlled by tuning the mass separator. The tuned beam, however, still contains a mixture of isobars. By recording the TOF data for events of interest in the experiment, one can select (tag) events from different regions of the TOF spectrum. In this way one could learn about isobaric effects on the reaction of interest. It is also remarkable that we were able to predict the level of ${ }^{132} \mathrm{Sb} /{ }^{132} \mathrm{Te}$ separation observed in the experiment.

\section{Conclusions}

It is well known that one can separate isobars in a mixed beam by exploiting the difference in energy loss for particles 


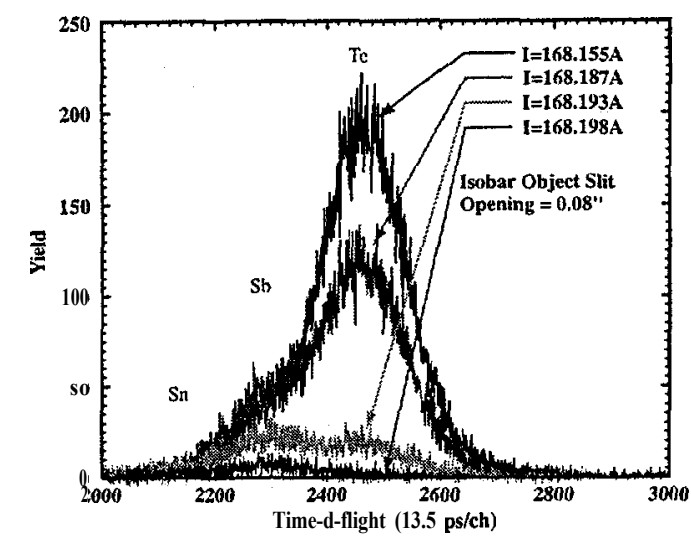

Fig. 9. Time-of-flight spectra for a mixture of ${ }^{132} \mathrm{Te},{ }^{132} \mathrm{Sb}$ and ${ }^{132} \mathrm{Sn}$ with different magnet settings.

different only in their nuclear charge $(z)$ that pass through an absorber. This difference in energy loss can be measured via TOF technique and allow isobar tagging or it can be used to select separate isobars with a magnetic separator, This method works best with energetic projectiles because the energy spread due to straggling decreases the closer the ion's charge state is to fully stripped. Most important for this method is keeping absorber inhomogeneities to a minimum, and for lower energy ions, the proposed gas cell is one possible method to accomplish this goal. In facilities where projectile fragmentation is used for RIB generation, the available energy will always be sufficient. However, unless isobars can be separated by some other method, it appears that in the design of an ISOL facility for the production of heavy RIBs, allowance must be made for boosting the projectile's energy to allow for isobar separation following energy loss in an absorber.

\section{REFERENCES}

[1] B. Rossi, High Energy Particles (Prentice-Hall, Engelwood Cliffs, 1961) 17 .

[2] J. F. Ziegler, program SRIM (http://www.research .ibm.com/ionbeams/\#SRIM).

[3] Program GEANT, PHYS431

(http://wwwinfo.cern.ch/asdoc/geant html3/node322.html).

[4] G. D. Mills, G. D. Alton, D. L. Haynes, and J. R. Beene, Physics Division Progress Report, ORNL-6957 (September 1998) (http://www.phy.ornl.gov/progress/hribf/randd/hri031.pdf).

[5] D. Bardayan et al., Phys. Rev. Lett. 83 (1999) 45.

[6] J. Gomez Del Campo et al., Phys. Rev. Lett. 86 (2001) 43.

[7] W. Starzecki, A. M. Stefanini, S. Lunardi, and C. Signorini, Nucl. Instr. and Meth. 193 (1982) 499.

[8] See list of beams on HRIBF website: (http://www.phy.ornl.gov/hribf/users/beams). 